Reprod. Nutr. Dévelop., 1986, 26 (2 A), 429-439.

\title{
Accumulation du monophosphate cyclique d'adénosine dans l'ovaire d'anguille (Anguilla anguilla L.) in vitro sous l'effet de la gonadotropine de carpe ou de la lutropine ovine : cinétique et thermodépendance
}

\author{
C. SALMON, J. MARCHELIDON, Elizabeth FONTAINE-BERTRAND, Y. A. \\ FONTAINE
}

Laboratoire de Physiologie générale et comparée du Muséum national d'Histoire naturelle, Laboratoire d'Endocrinologie comparée associé au C.N.R.S., 7, rue Cuvier, 75231, Paris, France.

Summary. Cyclic adenosine monophosphate accumulation in the eel (Anguilla anguilla L.) ovary in vitro under stimulation by carp gonadotropin or ovine lutropin : kinetics and thermodependence.

Cyclic AMP (cAMP) in pieces of eel ovary was greatly increased in vitro by the gonadotropin (CGTH) of carp, another teleost fish; after one hour at $20^{\circ} \mathrm{C}$, maximal stimulation $=31.7$ and E.D. $50=0.08 \mu \mathrm{g} / \mathrm{ml}$. Ovine lutropin (OLH) had less effect (maximal stimulation : 2.35 ; E.D. $50: 1.42 \mu \mathrm{g} / \mathrm{ml}$ ) ; its action suggested that it involved a subfraction (oLH/cGTH RAc) of the receptor-adenylate cyclase (RAc) systems which mediate the action of $c G T H$. Another difference was the percentage of total cAMP accumulated under hormonal stimulation and released into the incubation medium; this percentage was much higher with oLH than with $\mathrm{CGTH}\left(47 \mathrm{vs} 8 \%\right.$ after one hour at $\left.20^{\circ} \mathrm{C}\right)$. This result might be explained by a localization of oLH/cGTH RAc in cells (theca ?) situated on the outside of the follicles and/or by a relative lack of CAMP binding proteins in the case of cAMP produced by oLH/cGTH RAc. Kinetic and thermodependence studies also disclosed hormone-dependent differences; at $5{ }^{\circ} \mathrm{C}$, cAMP concentration was maximal after $40 \mathrm{~min}$ with oLH, whereas it was still increasing after $3 \mathrm{~h}$ with cGTH. Differences in the properties of phosphodiesterases and/or in the clearance rate of hormone-receptor (HR) complexes could explain these results.

The set of RAc systems in eel ovary recognizing fish gonadotropin would then be heterogeneous ; some of them would be endowed with original properties concerning receptor specificity and cAMP diffusion as well as associated phosphodiesterase activity and/or HR metabolism.

We suggest that at a stage of evolution when a single sensu stricto GTH is present (instead of two in tetrapods), " isoreceptors ", differing in specificity and in their fate after hormone binding, could be an important element in the fine regulation of gonadal functions.

Abréviations : adénosine $3^{\prime}-5^{\prime}$ monophosphate cyclique : AMPc ; adénylate cyclase : AC; lutropine ovine : oLH ; choriogonadotropine humaine: hCG; gonadotropine: GTH; gonadotropine de carpe : cGTH : récepteur : $\mathbf{R}$. 


\section{Introduction.}

Comme la lutropine et la follitropine chez les mammifères, la seule gonadotropine glycoprotéique présente chez les poissons téléostéens est capable de stimuler spécifiquement l'adénylate cyclase et l'accumulation de monophosphate cyclique d'adénosine dans l'ovaire (Fontaine et al., 1970 ; Idler et al., 1975 ; Fontaine-Bertrand et al., 1978). Dans tous les cas existent donc des récepteurs reconnaissant l'hormone physiologiquement compétente et couplés à l'adénylate cyclase. Au cours de l'évolution des vertébrés, gonadotropine et récepteurs se sont modifiés ce qui se manifeste par l'existence d'une spécificité zoologique d'action : une GTH d'une espèce donnée n'exerce souvent qu'un effet limité sur les paramètres de l'activité des gonades chez des receveurs phylogénétiquement éloignés (Fontaine, 1980). C'est ainsi que la lutropine ovine est beaucoup moins active que la gonadotropine de carpe (Cyprinus carpio L.) sur l'accumulation d'AMPc dans l'ovaire d'un autre poisson téléostéen, l'anguille (Anguilla anguilla L.) (Fontaine-Bertrand et al., 1981). Les expériences décrites ici visent à préciser les caractères de cette différence et à en analyser les mécanismes. Nous nous sommes, en particulier, intéressés à la cinétique de l'action hormonale - dont certaines données (Fontaine-Bertrand et al., 1981) suggéraient qu'elle n'était pas la même avec la cGTH et la oLH - et à sa thermodépendance ; on sait en effet que les hormones hypophysaires de poissons (poïkilothermes) et de mammifères (homéothermes) se comportent souvent, à divers point de vue, de façon différente selon la température (Fontaine, 1969 ; Marchelidon et al., 1979). Certains résultats préliminaires ont été précédemment présentés (Fontaine et al., 1985).

\section{Matériel et méthodes.}

Les anguilles argentées (240 à $340 \mathrm{~g})$, pêchées au filet dans les étangs de Péronne (Somme, France), sont gardées au laboratoire dans des bacs en eau courante dont la température est $12^{\circ} \pm 4^{\circ} \mathrm{C}$. Le rapport gonadosomatique (RGS = poids ovarien en grammes pour $100 \mathrm{~g}$ de poids corporel) est 1,3 à 1,8.

Dès le sacrifice (par décapitation), les ovaires sont prélevés et pesés dans du milieu d'incubation: tampon Krebs-Henseleit bicarbonate (concentration en

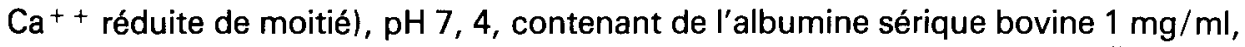
du glucose $1 \mathrm{mg} / \mathrm{ml}$ et de la théophylline $20 \mathrm{mM}$. Leur portion médiane est coupée en fragments de 20 à $30 \mathrm{mg}$; ceux-ci sont répartis deux par deux (provenant respectivement des ovaires droit et gauche) dans des flacons contenant $2 \mathrm{ml}$ de milieu et préincubés durant $20 \mathrm{~min}$ à la température désirée $(5,15$, 20 ou $25^{\circ} \mathrm{C}$ selon les expériences). Les fragments contenus dans chaque flacon sont ensuite transférés dans $2 \mathrm{ml}$ de milieu frais avec ou sans hormone et incubés de 4 à $180 \mathrm{~min}$ (cinétiques) ou pendant $60 \mathrm{~min}$ (étude des relations dose-réponse). Chaque incubation est réalisée en double.

La cGTH (Burzawa-Gérard, 1971) a été fournie par E. Burzawa-Gérard (Muséum, Paris) et la oLH par M. Jutisz (C.N.R.S, Gif-sur-Yvette). L'AMPc est extrait du tissu par la méthode précédemment décrite (Fontaine-Bertand et al., 1978) et dosé, en double, sur un aliquot de $50 \mu$ l d'extrait tissulaire ; dans le cas 
du milieu d'incubation, le dosage est effectué sur des aliquots de $150 \mu \mathrm{l}$. Le nucléotide est déterminé par compétition (Gilman, 1970 ; Tovey et al., 1974) avec une quantité connue d'AMPc- ${ }^{3} \mathrm{H}$ (CEA) pour une protéine de liaison du muscle de lapin (Walsh et al., 1968). A la fin de l'incubation, I'AMPc libre est éliminé par adsorption sur du charbon actif dont la concentration a été augmentée pour les mesures dans le milieu ( $36 \mathrm{mg} / \mathrm{ml}$ contre $4 \mathrm{mg} / \mathrm{ml}$ pour les extraits tissulaires) afin d'éviter les interférences dues aux sels du tampon.

Les résultats sont exprimés en picomole d'AMPc par mg d'ovaire et l'AMPc total représente la somme de l'AMPc du tissu et de l'AMPc sécrété dans le milieu d'incubation. La concentration hormonale donnant la moitié de la stimulation maximale (E.D. 50) est estimée graphiquement sur les courbes log(dose)-réponse et la stimulation maximale (Smax) est calculée par rapport aux témoins; les valeurs provenant de plusieurs expériences sont présentées sous la forme: moyenne \pm erreur standard de la moyenne. La comparaison des pourcentages de libération du nucléotide sous l'influence des deux hormones a été réalisée par analyse de covariance.

\section{Résultats.}

1) Effets de la cGTH et de la oLH a $20^{\circ} \mathrm{C}$.

a) Influence de la concentration hormonale sur I'AMPC tissulaire. - Les courbes représentant la concentration d'AMPc tissulaire (mesurée après une heure d'incubation) en fonction du logarithme de la concentration hormonale dans le milieu d'incubation sont d'allure sigmoïde et peuvent être caractérisées par E.D. 50 et Smax. Les moyennes de ces deux paramètres, déterminés sur de nombreuses expériences, sont respectivement $0,08 \pm 0,01 \mu \mathrm{g} / \mathrm{ml}$ et $31,7 \pm 2,9$ ( $\mathrm{n}=15$ ) pour la $\mathrm{cGTH}, 1,42 \pm 0,17 \mu \mathrm{g} / \mathrm{ml}$ et $2,35 \pm 0,12(\mathrm{n}=6$ ) pour la oLH. Les concentrations minimales produisant une augmentation significative de I'AMPc tissulaire sont, de façon reproductible, voisines de $1,3 \mathrm{ng} / \mathrm{ml}$ pour la cGTH et $800 \mathrm{ng} / \mathrm{ml}$ pour la oLH.

b) Mécanismes de l'action de la oLH. - L'effet de la oLH n'est pas aspécifique car la prolactine ovine et la corticotropine bovine n'exercent aucune action à des concentrations allant respectivement jusqu'à $100 \mu \mathrm{g} / \mathrm{ml}$ et $15 \mu \mathrm{g} / \mathrm{ml}$ (données non présentées). Nous avons cherché à savoir si la oLH était capable d'inhiber l'action de la cGTH. La figure 1 montre qu'il n'en est rien ; le seul effet significatif de la oLH (à une concentration induisant la Smax) est une faible augmentation de l'action d'une concentration faible de $\mathrm{cGTH}$, correspondant à une simple additivité.

c) Cinétique de l'action hormonale: AMPc dans le tissu et dans le milieu d'incubation. - Dans une première série d'expériences nous avons comparé les courbes $\log$ (concentration hormonale)-AMPc tissulaire obtenues après $1 \mathrm{~h}$ (cf 1a) et 20 min d'incubation. La Smax augmente nettement pour la cGTH entre $20 \mathrm{~min}$ 


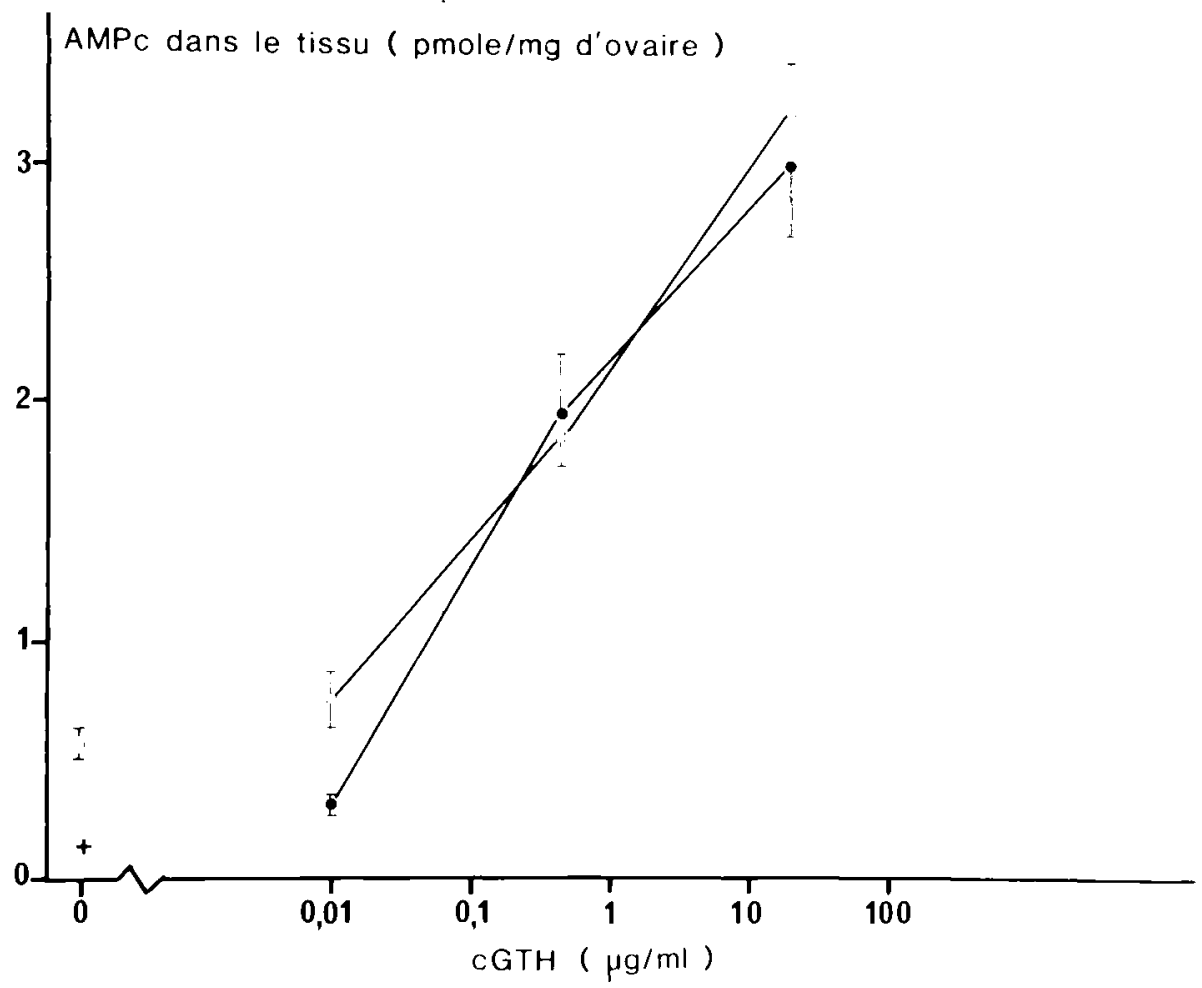

FIG. 1. - Absence d'inhibition par la $O L H$ de l'effet de la cGTH sur l'accumulation d'AMPC dans l'ovaire d'une anguille (poids du corps : $270 \mathrm{~g}$; RGS : 1,68). Les incubations sont effectuées à $20{ }^{\circ} \mathrm{C}$.

Chaque point représente la moyenne de deux déterminations : + 40 min sans hormone ; 20 min sans hormone puis $20 \mathrm{~min}$ en présence de cGTH ; $20 \mathrm{~min}$ en présence de oLH $(20 \mu \mathrm{g} / \mathrm{ml})$ puis $20 \mathrm{~min}$ en présence de oLH $+\mathrm{CGTH} ; 040 \mathrm{~min}$ en présence de oLH $(20 \mu \mathrm{g} / \mathrm{ml})$. I intervalle entre les deux déterminations.

(òu elle est de $21,3 \pm 3,0$ ) et $60 \mathrm{~min}$; par contre elle tendrait plutôt à diminuer dans le cas de la oLH (Smax après $20 \mathrm{~min}=2,52 \pm 0,31$ ). Une sortie d'AMPc dans le milieu d'incubation, plus importante avec la oLH qu'avec la cGTH pouvait expliquer ces résultats. Les expériences qui vont être décrites ont confirmé cette hypothèse. Nous avons comparé les cinétiques d'accumulation de l'AMPc dans le tissu et dans le milieu, d'une part avec une concentration de oLH déterminant la Smax $(20 \mu \mathrm{g} / \mathrm{ml})$, d'autre part avec une concentration de cGTH $(0,01 \mu \mathrm{g} / \mathrm{ml})$ qui exerce, pour des incubations courtes $(4 \mathrm{~min})$, le même effet que $20 \mu \mathrm{g} \mathrm{oLH} / \mathrm{ml}$ sur l'AMPc tissulaire. A partir de 20 min, l'AMPc tissulaire devient beaucoup plus important avec la cGTH qu'avec la oLH mais la quantité libérée dans le milieu est, à tous les temps étudiés (jusqu'à 3 h) 2 à 3 fois supérieure avec la oLH. C'est dire que la quantité d'AMPc dans le milieu, exprimée en pourcentage de l'AMPc total, est toujours plus élevée avec la oLH qu'avec la cGTH. L'évolution de ce pourcentage en fonction du temps est présenté figure 2 ; on observe que la sortie de 
I'AMPc dans le milieu, après une décharge initiale puis une stabilisation, s'établit dès $10 \mathrm{~min}$ avec la oLH mais ne commence vraiment qu'après 60 min dans le cas de la cGTH. Enfin les résultats de nombreuses expériences effectuées avec différentes concentrations hormonales (la durée de l'incubation étant de une heure) montrent que l'AMPc du milieu est proportionnel à l'AMPc total d'une part pour la $\mathrm{cGTH}$ (AMPc milieu $=0,086$ AMPc total $+0,009, r=0,89$, d.l. $=84$, $\mathrm{p}<0,01$ ), d'autre part pour la oLH (AMPc milieu $=0,47$ AMPc total $-0,007$, $r=0,61$ d.I. $=31, p<0,01)$. Le pourcentage de l'AMPc total présent dans le milieu ne dépend donc pas du niveau de la stimulation hormonale mais il dépend considérablement de la nature de l'hormone, passant (pour une incubation de une heure) de 8,6 avec la cGTH à 47 avec la oLH $(F=20,3$, d.l. $=1$ et 115 , $p<0,05)$.

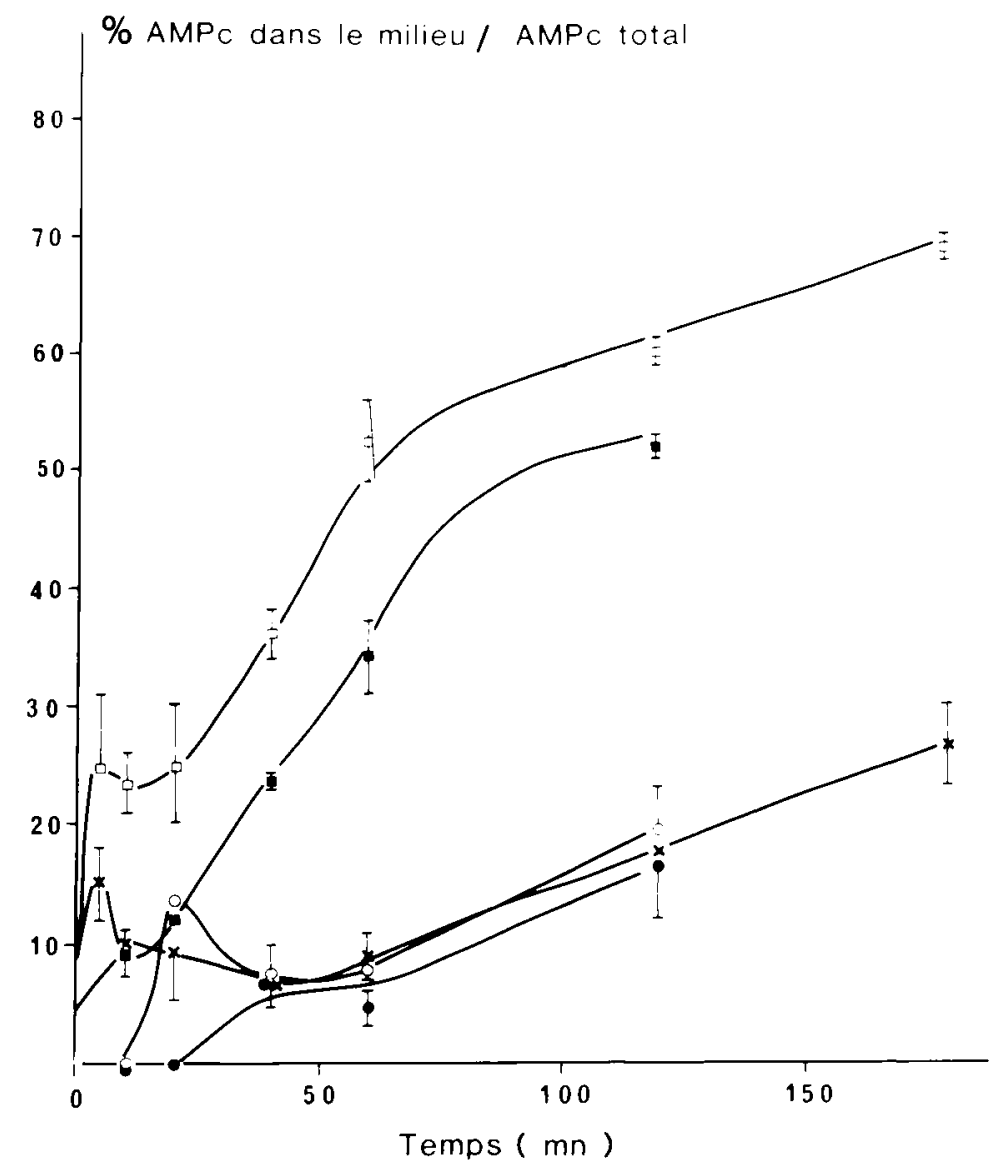

FIG. 2 - Evolution du pourcentage d'AMPc dans le milieu d'incubation par rapport à l'AMPC total en fonction du temps d'incubation : effets de la $c G T H$ et de la oLH. Les incubations sont effectuées à $20^{\circ} \mathrm{C}$.

Chaque point représente la moyenne de deux déterminations : $\bullet \mathrm{cGTH} 0,002 \mu \mathrm{g} / \mathrm{ml} ; \times \bigcirc \mathrm{cGTH}$ $0,01 \mu \mathrm{g} / \mathrm{ml} ; \square \quad$ oLH $20 \mu \mathrm{g} / \mathrm{ml}$ (2 expériences). I intervalle entre les deux déterminations. 
2) Thermodépendance comparée des effets de la cGTH et de la oLH.

Nous avons suivi l'accumulation d'AMPc dans le tissu et dans le milieu à 5 , 15 et $25^{\circ} \mathrm{C}$ sous trois types de stimulation hormonale : oLH $20 \mu \mathrm{g} / \mathrm{ml}$, cGTH 0,01 $\mu \mathrm{g} / \mathrm{ml}$ et $\mathrm{cGTH} 1 \mu \mathrm{g} / \mathrm{ml}$ (concentration déterminant une stimulation maximale ou quasi maximale).

a) Accumulation d'AMPc total. - Dans le cas de la oLH (fig. 3) la quantité totale d'AMPc accumulée est, à tous les temps, plus élevée quand la température

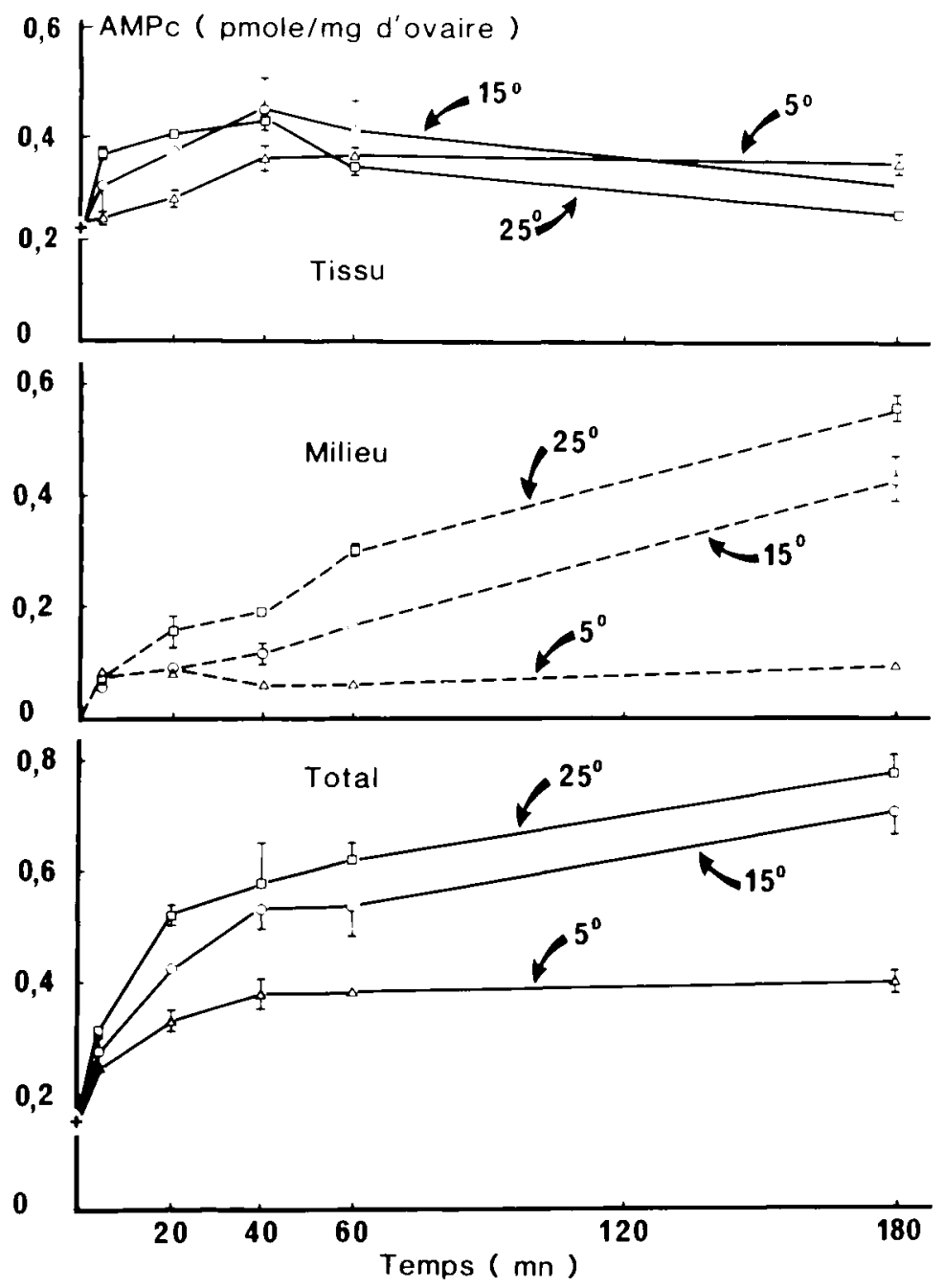

FIG. 3. - Thermodépendance de la cinétique d'accumulation de l'AMPC dans l'ovaire d'une anguille (poids du corps : $290 \mathrm{~g}$; RGS : 1,43) en présence de oLH $(20 \mu \mathrm{g} / \mathrm{ml}$ ). Températures d'incubation : $5^{\circ} \mathrm{C}(\triangle), 15^{\circ} \mathrm{C}(0), 25^{\circ} \mathrm{C}(\square)$. Chaque point représente la moyenne de deux déterminations. $\perp$ intervalle entre les deux déterminations. Chez les témoins, les concentrations en AMPc dans le tissu sont $0,25 \mathrm{pmole} / \mathrm{mg}$ au temps 0 et $0,16,0,18,0,17 \mathrm{pmole} / \mathrm{mg}$ après 180 min d'incubation à 5,15 et $25^{\circ} \mathrm{C}$ respectivement ; pour l'AMPc total, les concentrations sont 0,21 0,25 et $0,40 \mathrm{pmole} / \mathrm{mg}$ à 5,15 et $25^{\circ} \mathrm{C}$ respectivement après $180 \mathrm{~min}$ d'incubation. 
est plus élevée. L'analyse des courbes montre que, initialement, la vitesse d'accumulation est nettement thermodépendante. Elle diminue ensuite rapidement, la quantité totale d'AMPc restant stable (à $5^{\circ}$ ) ou continuant à s'élever légèrement (à 15 et $25^{\circ} \mathrm{C}$ ).

Avec la concentration faible de cGTH (fig. 4) la vitesse d'accumulation est $d^{\prime}$ abord plus élevée à 15 et $25^{\circ} \mathrm{C}$ qu'à $5^{\circ} \mathrm{C}$. Sa diminution ultérieure est plus tar-

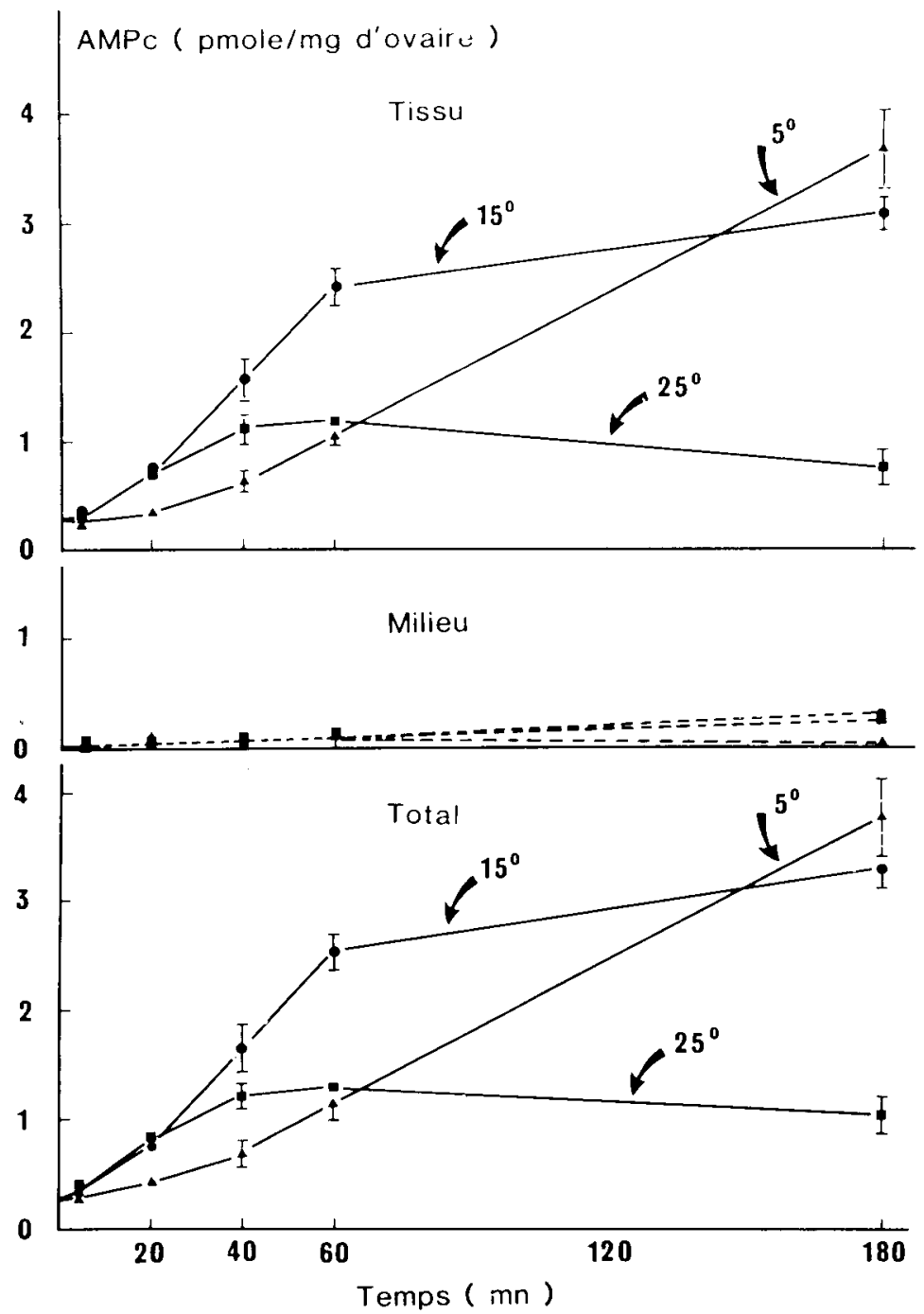

FIG. 4 - Thermodépendance de la cinétique d'accumulation de l'AMPC dans l'ovaire d'une anguille (poids du corps : $254 \mathrm{~g}$; RGS : 1,51) en présence de $c G T H(0,01 \mu \mathrm{g} / \mathrm{ml}$ ). Températures d'incubation : $5{ }^{\circ} \mathrm{C}(\boldsymbol{\Delta}), 15^{\circ} \mathrm{C}(\bullet), 25^{\circ} \mathrm{C}(\boldsymbol{\square})$. Chaque point représente la moyenne de deux déterminations. I Intervalle entre les deux déterminations. Chez les témoins, les concentrations en AMPc dans le tissu sont $0,30 \mathrm{pmole} / \mathrm{mg}$ au temps 0 et $0,21,0,20,0,23 \mathrm{pmole} / \mathrm{mg}$ après $180 \mathrm{~min}$ d'incubation à 5,15 et $25{ }^{\circ} \mathrm{C}$ respectivement ; pour l'AMPc total, les concentrations sont $0,26,0,26$ et $0,35 \mathrm{pmole} / \mathrm{mg}$ à 5,15 et $25^{\circ} \mathrm{C}$ respectivement après $180 \mathrm{~min}$ d'incubation. 
dive qu'avec la oLH, d'autant plus tardive (et d'autant moins marquée) que la température est plus basse, si bien qu'après trois heures d'incubation la quantité d'AMPc est beaucoup plus faible à $25^{\circ} \mathrm{C}$ qu'à 15 et $5^{\circ} \mathrm{C}$. Avec la concentration forte de cGTH la vitesse maximale est, aux trois températures, atteinte plus rapidement qu'avec $0,01 \mu \mathrm{g} / \mathrm{ml}$ de cGTH. Après cet optimum, les cinétiques présentent des similitudes quelle que soit la dose d'hormone; cependant, avec $1 \mu \mathrm{g}$ $\mathrm{cGTH} / \mathrm{ml}$, I'AMPc s'élève moins $\left(5^{\circ} \mathrm{C}\right)$ ou diminue plus $\left(25^{\circ} \mathrm{C}\right)$ qu'avec $0,01 \mu \mathrm{g}$ $\mathrm{cGTH} / \mathrm{ml}$.

b) Sortie de l'AMPc dans le milieu d'incubation. - Ce phénomène est dans tous les cas très thermodépendant (fig. 3 et 4). A $5^{\circ} \mathrm{C}$, la quantité d'AMPc présente dans le milieu est très faible et ne s'élève pas nettement, au cours de l'incubation, après la faible décharge initiale. Aux températures supérieures, le pourcentage de l'AMPc total présent dans le milieu augmente avec la durée d'incubation et avec la température ; il est toujours plus élevé en présence de oLH qu'en présence de cGTH laprès $1 \mathrm{~h}$ d'incubation à 15 ou $25^{\circ} \mathrm{C}$, il est respectivement égal à 31 et $49 \%$ avec la oLH, 4 et $9 \%$ avec la cGTH $0,01 \mu \mathrm{g} / \mathrm{ml}, 5$ et $11 \%$ avec la $\mathrm{cGTH} 1 \mu \mathrm{g} / \mathrm{ml}$ ).

\section{Discussion.}

La oLH stimule de façon faible mais nette la production d'AMPc par des fragments d'ovaire d'anguille (Fontaine-Bertrand et al., 1981, Fontaine et al., 1985 et cette note) ; il en est de même pour la gonadotropine chorionique humaine (hCG) (Salmon et al., 1985). Plusieurs faits (Salmon et al., 1985) conduisent à supposer que ces hormones agissent par l'intermédiaire de systèmes récepteur-adénylate cyclase mis aussi en jeu par la GTH de poisson. En particulier, nous avons pu mettre en évidence une désensibilisation croisée entre la cGTH et la hCG (Salmon et al., 1985). L'absence, observée ici, d'additivité des effets de doses élevées de oLH et de cGTH va dans le même sens ; elle n'est pourtant pas décisive car la stimulation maximale par la oLH est très inférieure à celle due à la cGTH. Nous ne pouvons donc pas complètement éliminer la possibilité qu'il existe, dans l'ovaire d'anguille, des systèmes RAc spécifiques des GTH mammaliennes; comme on peut néanmoins se demander quelle serait la signification physiologique de ces derniers, nous supposerons ici que les gonadotropines de mammifères et de poissons partagent les mêmes RAc.

Pourquoi, dans ces conditions, l'action de la oLH est-elle si faible comparée à celle de la cGTH ? La différence des E.D. 50 traduit sans doute une différence des affinités des liaisons hormone-récepteur (Fontaine-Bertrand et al., 1981 ; Fontaine et al., 1985). Nous nous attacherons plus, ici, à la différence des Smax. Pour l'expliquer, une première hypothèse est que la oLH ne met en jeu qu'une partie faible des systèmes récepteur-adénylate cyclase de la cGTH (RAc cGTH); la seconde est que la oLH met en jeu tous les RAc mais que la stimulation de I'adénylate cyclase qui en résulte est, pour chaque complexe LH-RAc, bien moindre que pour un complexe cGTH-RAc. Dans cette éventualité, la oLH devrait être 
capable de se lier à tous les RAc et de diminuer l'action de la cGTH ajoutée ultérieurement. Ceci n'est pas observé expérimentalement et nous supposons donc plutôt que seule une fraction des RAc est mise en jeu par la oLH; ce sousensemble, que nous appellerons RAc cGTH/oLH, se caractériserait donc, vis-à-vis des autres RAc, par une moindre spécificité de reconnaissance des récepteurs (ceux-ci reconnaissant non seulement la cGTH mais aussi la oLH).

Les RAc cGTH/oLH présentent des propriétés originales dans des domaines tout différents :

a) La libération d'AMPc dans le milieu. Le pourcentage de I'AMPc qui sort (sans doute de façon passive, Selstam et al., 1976) dans le milieu est considérablement plus élevé avec la oLH (et la hCG, Salmon et al., 1985) qu'avec la cGTH, ce qui peut refléter une moindre liaison à des protéines intracellulaires ou une localisation particulière des RAc cGTH/oLH. Dans l'ovaire de rat, la libération d'AMPc est plus importante avec la oLH qu'avec la oFSH (Selstam et al., 1976) ; comme la première agit surtout sur les cellules de la thèque et la seconde sur celles plus internes de la granulosa (Richards, 1980), il est tentant, par analogie, de situer, chez l'anguille, les RAc cGTH/oLH dans les cellules (thécales ?) de la périphérie des follicules ovariens ;

b) La cinétique de l'accumulation d'AMPc. C'est ainsi qu'à $5^{\circ} \mathrm{C}$, la quantité d'AMPc produite est maximale dès 40 minutes en présence de oLH tandis qu'elle augmente jusqu'à au moins $3 \mathrm{~h}$ avec la cGTH, de façon dépendante de la dose. Ceci suggère deux hypothèses non exclusives: dégradation plus rapide ou synthèse moins durable du nucléotide au niveau des RAc cGTH/oLH par rapport aux autres RAc. La première impliquerait que l'AMPc sensible à la oLH soit soumis à une activité phosphodiestérase particulièrement importante (l'addition de théophylline peut ne pas inhiber entièrement cette activité comme le montrent les résultats obtenus à $25{ }^{\circ} \mathrm{C}$ en présence de $\mathrm{CGTH}$ ), la seconde que les complexes oLH-R soient moins stables ou s'éliminent plus rapidement que les complexes cGTH-R ; ceci serait en accord avec la différence des E.D. 50 pour la cGTH et la oLH puisque ces paramètres reflètent vraisemblablement les $\mathrm{Kd}$ respectifs.

c) La thermodépendance des phénomènes. L'accumulation de I'AMPc est plus thermodépendante en présence de cGTH que de oLH. Les temps mis pour atteindre la vitesse maximale d'accumulation ainsi que l'évolution ultérieure de cette vitesse semblent peu sensibles à la température dans le cas de la lutropine. Ceci peut refléter la sortie plus importante de I'AMPc dans le milieu en présence de oLH, AMPc qui est donc soustrait à l'activité phosphodiestérasique intracellulaire et à ses variations en fonction de la température. On ne peut exclure, non plus, que la stabilité thermique des complexes $\mathrm{H}-\mathrm{R}$ dépende de la nature de l'hormone, la disparition des complexes oLH-R étant moins thermodépendante que celle des complexes cGTH-R.

Quelle est l'importance relative des RAc cGTH/oLH dans l'ensemble des RAc cGTH ? Si on l'estime par le rapport des stimulations maximales déterminées pour la oLH et la cGTH à $5{ }^{\circ} \mathrm{C}$, on obtient une valeur d'environ $8 \%$; ce chiffre est sensiblement plus faible que celui, obtenu par des expériences de désensibilisation croisée entre cGTH et hCG (Salmon et al., 1985), qui dépasse $20 \%$. La première 
estimation est effectivement erronée (même dans le cas où LH et hCG ont exactement les mêmes récepteurs) si la Smax atteinte en présence de cGTH est différente dans les deux catégories de RAc ou bien si la cGTH et une gonadotropine mammalienne déterminent des Smax différentes au niveau de chaque RAc cGTH/OLH.

L'ensemble des résultats présentés ici suggère qu'une partie des RAc cGTH de l'ovaire d'anguille se singularise. Ce sous-ensemble (Rac cGTH/oLH) est susceptible d'avoir une localisation et des fonctions particulières. Or ces RAc cGTH/oLH ont d'abord été caractérisés par une tout autre propriété : une relativement faible spécificité de reconnaissance des récepteurs (mise en évidence de façon pharmacologique grâce à l'utilisation d'une hormone mammalienne). Quelle peut être la signification physiologique de cette dernière propriété ? Nous proposons, à titre d'hypothèse de travail, que ces récepteurs sont également capables de se singulariser, vis-à-vis des autres récepteurs de la cGTH, quant aux modalités de leur liaison avec l'hormone endogène. Une telle situation pourrait jouer un rôle dans la régulation fine des gonades des poissons en permettant un contrôle relativement indépendant de diverses fonctions par une seule gonadotropine. D'un point de vue évolutif, l'existence, chez les poissons téléostéens d'« isorécepteurs " (avec des propriétés différentes) pour la GTH unique aurait alors précédé, et peut-être favorisé, chez les tétrapodes (Fontaine, 1980), l'apparition et la différenciation ultérieure de la seconde GTH, de type FSH.

Reçu en septembre 1985. Accepté en novembre 1985.

\section{Références}

BURZAWA-GÉRARD E., 1971. Purification d'une hormone gonadotrope hypophysaire de poisson téléostéen, la carpe (Cvprinus carpio L.) Biochimie, 53, 545-552.

FONTAINE Y. A., 1969. La spécificité zoologique des protéines hypophysaires capables de stimuler la thyroïde, Acta endoc., 60, suppl. 136, 1-154.

FONTAINE Y. A., 1980. Les hormones gonadotropes de l'hypophyse : Biochimie et biologie comparées ; spécificité et évolution Reprod. Nutr. Dévelop., 20, 381-418.

FONTAINE Y. A., BURZAWA-GÉRARD E., DELERUE-LE BELLE N., 1970. Stimulation hormonale de l'activité adényl-cyclasique de l'ovaire d'un Poisson Téléostéen, Le Cyprin (Carassius auratus L.I, C.R. Acad. Sci., Paris Série D, 271, 780-783.

FONTAINE Y. A., SALMON C., FONTAINE-BERTRAND E., MARCHELIDON J., 1985. The mechanism and the specificity of action of pituitary gonatropins 169-172. In LOFTS B., HOLMES W. N., Current trends in comparative endocrinology. Hong Kong Univ. Press, Hong Kong.

FONTAINE-BERTRAND E., MARCHELIDON J., SALMON C., FONTAINE Y. A., 1981. Les sousunités des lutropines : une activité gonadotrope intrinsèque de $\alpha$ et le rôle de $\beta$ dans le déterminisme de la spécificité zoologique d'action, C.R. Acad. Sci, Paris Sér. III, 292, 507-510. 
FONTAINE-BERTRAND E., SALMON C., FONTAINE Y. A., 1978. Effet d'hormones gonadotropes, in vitro, sur la concentration de l'adénosine monophosphate cyclique dans l'ovaire de l'anguille (Anguilla anguilla L.), Ann. Biol. anim. Bioch. Biophys., 18, 805-811.

GILMAN A. G., 1970. A protein binding assay for adenosine $3^{\prime} 5^{\prime}$ cyclic monophosphate, Proc. nat. Acad. Sci., 67, 305-312.

IDLER D. R., HWANG S. J., BAZAR L. S., 1975. Fish gonadotropin(s). I. Bioassay of salmon gonadotropin(s) in vitro with immature trout gonads, Endocr. Res. Commun., 2, 199-213.

MARCHELIDON J., SALESSE R., GARNIER J., BURZAWA-GÉRARD E., FONTAINE Y. A., 1979. Zoological origin of gonadotropin subunits and association kinetics, Nature, 281, 314-315.

RICHARDS J. S., 1980. Maturation of ovarian follicles: Actions and interactions of pituitary and ovarian hormones on follicular cell differentiation, Physiol. Rev., 60, 51-89.

SALMON C., MARCHELIDON J., FONTAINE-BERTRAND E., FONTAINE Y. A., 1985. Human chorionic gonadotropin and immature fish ovary: Characterization and mechanism of the in vitro stimulation of cyclic adenosine monophosphate accumulation, Gen. comp. Endocrinol., 58, 101-108.

SELSTAM G., ROSBERG S., LILJEKVIST J., GRÖNQUIST L., PERKLEV T., AHREN K., 1976. Differences in action of $\mathrm{LH}$ and FSH on the formation of cyclic AMP in the prepubertal rat ovary, Acta endoc., 81, 150-164.

TOVEY K. C., OLDHAM K. G., WHELAN J. A. M., 1974. A simple direct assay for cyclic AMP in plasma and other biological samples using an improved competitive protein binding technique, Clin. chim. Acta, 56, 221-234.

WALSH D. A., PERKINS J. P., KREBS E. G., 1968. An adenosine 3',5'-monophophastedependent protein kinase from rabbit skeletal muscle, J. biol. Chem., 243, 3763-3771. 